\title{
Impact of left atrial appendage exclusion on left atrial function
}

\author{
Keiji Kamohara, MD, ${ }^{a}$ Zoran B. Popović, MD, ${ }^{\text {b }}$ Masao Daimon, MD, ${ }^{\text {b }}$ Maureen Martin, RDCS, ${ }^{\text {b }}$ Yoshio Ootaki, MD, PhD, \\ Masatoshi Akiyama, MD, PhD, ${ }^{a}$ Firas Zahr, MD, ${ }^{a}$ Faruk Cingoz, MD, ${ }^{a}$ Chiyo Ootaki, MD, ${ }^{a}$ Michael W. Kopcak, Jr, BA, \\ Raymond Dessoffy, AA, ${ }^{a}$ Jenny Liu, BA, ${ }^{a}$ James D. Thomas, MD, ${ }^{b}$. Marc Gillinov, MD, ${ }^{c}$ and \\ Kiyotaka Fukamachi, MD, PhDa
}

From the Department of Biomedical Engineering, Lerner Research Institute, ${ }^{a}$ and the Departments of Cardiovascular Medicine ${ }^{\mathrm{b}}$ and Thoracic and Cardiovascular Surgery, ${ }^{\mathrm{C}}$ The Cleveland Clinic, Cleveland Ohio.

Supported by the Atrial Fibrillation Innovation Center, an Ohio Wright Center of Innovation, and by AtriCure, Inc, Cincinnati, Ohio. A.M.G. is a consultant to AtriCure, Inc. The Cleveland Clinic has an indirect interest in AtriCure, Inc, through its interest in a private fund that has an equity interest in AtriCure, Inc.

Received for publication May 4, 2006; revisions received Aug 10, 2006; accepted for publication Aug 25, 2006.

Address for reprints: Kiyotaka Fukamachi, $\mathrm{MD}, \mathrm{PhD}$, Department of Biomedical Engineering/ND20, Cleveland Clinic, 9500 Euclid Ave, Cleveland, OH 44195 (E-mail: fukamak@ccf.org).

J Thorac Cardiovasc Surg 2007;133:174-81

$0022-5223 / \$ 32.00$

Copyright (๑) 2007 by The American Association for Thoracic Surgery

doi:10.1016/j.jtcvs.2006.08.057
Objectives: We sought to investigate the short-term and midterm effects of left atrial appendage exclusion on left atrial function. Left atrial appendage exclusion is considered a possible therapeutic option for stroke prevention in patients with atrial fibrillation. Favorable outcomes have encouraged widespread use of left atrial appendage exclusion for cardiac surgical patients-even for patients in sinus rhythm who have stroke risk factors; however, the chronic effects on left atrial function of left atrial appendage exclusion are unclear.

Methods: Nineteen mongrel dogs $(29.7 \pm 5.2 \mathrm{~kg})$ in sinus rhythm were studied. The Doppler signals from the pulmonary venous flow, transmitral flow, and tissue Doppler imaging of mitral annular motion were obtained before and after left atrial appendage exclusion. Dogs were evaluated in the same manner at 7 days $(\mathrm{n}=2)$, 30 days $(\mathrm{n}=7)$, or 90 days $(\mathrm{n}=10)$ after left atrial appendage exclusion.

Results: Except for a significant increase in early diastolic transmitral flow velocity after left atrial appendage exclusion $(P=.01)$, no significant differences were found in any parameters related to the transmitral flow and tissue Doppler imaging measurements throughout follow-up. The systolic components of pulmonary venous flow at follow-up revealed a significant reduction relative to baseline (peak systolic velocity $P<.0001$, systolic velocity-time integral $P<.0001$ ), despite the lack of significant changes in left atrial pressure, left ventricular volume, and stroke volume.

Conclusion: Left atrial appendage exclusion may affect left atrial reservoir function in the short-term and midterm periods. Further long-term studies with more clinically relevant models are needed.

A trial fibrillation (AF) is a major risk factor for stroke, a common cause of serious disability and death. ${ }^{1,2}$ Congestive heart failure is often complicated by $\mathrm{AF}$ and promotes the development of $\mathrm{AF}$ even in patients with sinus rhythm. ${ }^{3,4}$ The left atrial appendage (LAA) is a major source of atrial thrombi in patients with AF. ${ }^{5,6}$ LAA exclusion has therefore been considered a potential therapeutic option for stroke prevention in patients with AF. Favorable outcomes of LAA exclusion in surgical patients have encouraged widespread use of this procedure, extending it to include patients in sinus rhythm who have stroke risk factors. ${ }^{7}$ Although some in vitro ${ }^{8}$ and in vivo ${ }^{9-11}$ studies have reported that LAA exclusion can alter left atrial (LA) compliance, the chronic impact of LAA exclusion on LA and left ventricular (LV) functions still remain unclear.

Recently, pulsed Doppler analysis of pulmonary venous (PV) flow has provided additional information about atrial filling and function, which has been used in conjunction with the transmitral flow spectra or tissue Doppler imaging (TDI) of mitral annular motion to enhance assessments of abnormalities of ventricular 


\author{
Abbreviations and Acronyms \\ $\mathrm{AF}=$ atrial fibrillation \\ $\mathrm{CO}=$ cardiac output \\ $\mathrm{EDV}=$ end-diastolic volume \\ $\mathrm{EE}=$ epicardial echocardiography \\ $\mathrm{EF}=$ ejection fraction \\ ESV $=$ end-systolic volume \\ $\mathrm{LA}=$ left atrium \\ LAA $=$ left atrial appendage \\ LAP $=$ left atrial pressure \\ $\mathrm{LV}=$ left ventricle \\ $\mathrm{PV}=$ pulmonary vein \\ $\mathrm{SV}=$ stroke volume \\ TDI $=$ tissue Doppler imaging \\ VTI $=$ velocity-time integral
}

filling. ${ }^{12-20}$ With the pulsed Doppler technique, the relationship between the PV flow pattern and dynamic changes of the LV and LA can be investigated under several different conditions in animals and human beings ${ }^{12-15}$ and therefore used to assess the changes in LA or LV diastolic function resulting from LAA exclusion.

\section{Materials and Methods}

Nineteen mongrel dogs $(29.7 \pm 5.2 \mathrm{~kg})$ in sinus rhythm were used in this study. This study was approved by the Cleveland Clinic's Institutional Animal Care and Use Committee, and all animals received humane care in compliance with the "Guide for the Care and Use of Laboratory Animals" (http://www.nap.edu/catalog/ 5140.html).

To evaluate the short-term and midterm effects of LAA exclusion on LA and LV functions in dogs, the LAA was excluded while the heart was beating with a novel exclusion device. ${ }^{21}$ Hemodynamic and echocardiographic measurements were performed before and after LAA exclusion. Dogs were then evaluated at 7 days $(n=2), 30$ days $(n=7)$, or 90 days $(n=10)$ after LAA exclusion in the same manner.

\section{Study Protocol and Surgical Procedures}

Initial study. All surgical procedures were performed under sterile conditions. The animals were anesthetized with intravenous thiopental $(20 \mathrm{mg} / \mathrm{kg})$ and ventilated through an endotracheal tube by a respirator (Servo Ventilator 900C; Siemens-Elema AB, Solna, Sweden). Anesthesia was maintained with isoflurane (0.5\%-2.5\%). The animals were placed in the right lateral position with electrocardiographic leads attached to the extremities. A left lateral neck incision was made to isolate the left carotid artery for arterial pressure monitoring.

A left thoracotomy was performed at the fourth intercostal space to obtain access to the heart. The pericardium was opened, and the heart was suspended in a pericardial cradle to expose the LAA. A 14-gauge angiocatheter was inserted into the left upper PV to monitor the LA pressure (LAP). With epicardial echocardiography (EE), 2-dimensional (2D) and all Doppler echocardio- graphic images were obtained. Hemodynamic data, including heart rate, systemic arterial pressure, and LAP, were also collected at baseline. Ventilatory support was transiently stopped during dataacquisition periods. Hemodynamic parameters were digitized in real time at a sampling rate of $200 \mathrm{~Hz}$ with a data-acquisition system (PowerLab; AD Instruments, Inc, Mountain View, Calif) and stored on a hard disk for subsequent analyses with a custommade visual basic program on Excel software (Excel 2000; Microsoft Corporation, Redmond, Wash).

After all baseline data were obtained, the LAA was occluded with a simple device that permitted LAA exclusion from the outer surface of the heart while the heart was beating. Blood flow into the LAA was assessed before and after the LAA exclusion by $2 \mathrm{D}$ EE. If the device was not precisely placed at the base of the LAA, the device was simply repositioned and reapplied in the proper location $^{21,22}$ for successful exclusion of the LAA. After LAA exclusion, echocardiographic and hemodynamic studies were repeated in the same manner. The interval between the baseline data acquisition and postexclusion data acquisition was less than 10 minutes for all animals. After closure of the pericardium, the chest was closed with a chest drainage tube in place. Animals were followed up for 7 days $(\mathrm{n}=2), 30$ days $(\mathrm{n}=7)$, or 90 days $(\mathrm{n}=$ 10). With the exception of analgesics and antibiotics, no additional medications were administered during the postoperative period.

Terminal study. On the day of the terminal study, animals were placed under general anesthesia according to the protocol of the initial study. A right inguinal incision was made to isolate the right femoral artery for continuous monitoring of arterial pressure. The chest was reopened from the original thoracotomy incision, and the pericardium was reopened to expose the LAA occlusion device. Hemodynamic assessment and EE were performed in the same manner as described for the initial study to evaluate the short-term and midterm effects of LAA exclusion on LA function.

\section{Echocardiographic Measurements}

Echocardiographic examinations were performed with a Sequoia 512 digital ultrasonographic system (Siemens, Mountain View, Calif) with a high-frequency transducer (frequency $5-7 \mathrm{MHz}$ ). All Doppler echocardiographic and TDI images were obtained and recorded during three to five consecutive cardiac cycles in sinus rhythm.

The LA body area was measured by planimetry in the apical 4-chamber view. The LA body volume was also assessed by a biplane area-length method from the apical 4- and 2-chamber views. It is true that the biplane long-axis method relies on various geometric assumptions. To assess the potential error introduced by our method in this animal series, we validated it against a criterion standard of 3-dimensional echocardiography ${ }^{23,24}$ with real-time 3-dimensional data obtained by Vivid 7 echocardiographic machine and Tomtec software (Tomtec Gmbh, Unterschlessheim, Germany). Bland-Altman analysis ${ }^{25}$ showed $95 \%$ limits of agreement of -2.4 and $+7.4 \mathrm{~mL}$, indicating that our method used for LA volume analyses in this animal series, although not perfect, was accurate enough to track changes in LA volumes. The LV end-diastolic volume (EDV) and end-systolic volume (ESV) were measured by single-plane Simpson rule. LV ejection fraction (EF) was calculated by the following equation: $100 \times(\mathrm{EDV}-\mathrm{ESV}) /$ EDV. The LV stroke volume (SV) was calculated as the difference 
TABLE 1. Hemodynamic and two-dimensional echocardiographic variables at baseline, after left atrial appendage exclusion, and at follow-up

\begin{tabular}{|c|c|c|c|c|c|c|}
\hline & \multicolumn{2}{|c|}{ Baseline } & \multicolumn{2}{|c|}{ After exclusion } & \multicolumn{2}{|c|}{ Follow-up } \\
\hline & $\mathbf{n}$ & Value & $\mathbf{n}$ & Value & $\mathbf{n}$ & Value \\
\hline \multicolumn{7}{|l|}{ Hemodynamics } \\
\hline Heart rate (beats/min) & 19 & $120 \pm 17$ & 19 & $124 \pm 19$ & 19 & $120 \pm 13$ \\
\hline Systolic BP (mm Hg) & 19 & $124 \pm 20$ & 19 & $118 \pm 16$ & 19 & $121 \pm 10$ \\
\hline Diastolic BP $(\mathrm{mm} \mathrm{Hg})$ & 19 & $88 \pm 19$ & 19 & $77 \pm 17$ & 19 & $79 \pm 16$ \\
\hline Mean LA pressure $(\mathrm{mm} \mathrm{Hg})$ & 19 & $8.8 \pm 3.4$ & 19 & $8.9 \pm 3.2$ & 19 & $8.1 \pm 3.5$ \\
\hline \multicolumn{7}{|c|}{ Two-dimensional epicardial echocardiography } \\
\hline Mitral regurgitation grade $\leq 1$ & 19 & 19 & 19 & 19 & 19 & 19 \\
\hline $\mathrm{LA}$ area $\left(\mathrm{cm}^{2}\right)$ & 19 & $11.0 \pm 3.1$ & 19 & $9.8 \pm 2.6$ & 19 & $9.6 \pm 2.5$ \\
\hline LA volume $\left(\mathrm{cm}^{3}\right)$ & 13 & $28.7 \pm 3.8$ & 10 & $23.8 \pm 5.4$ & 13 & $24.2 \pm 5.7$ \\
\hline End-diastolic volume (mL) & 17 & $63.5 \pm 19.4$ & 18 & $59.7 \pm 20.7$ & 19 & $59.3 \pm 18.9$ \\
\hline End-systolic volume (mL) & 16 & $25.6 \pm 11.9$ & 18 & $26.8 \pm 14.4$ & 19 & $26.8 \pm 12.6$ \\
\hline Stroke volume (mL) & 16 & $37.8 \pm 13.7$ & 18 & $32.8 \pm 12.4$ & 18 & $32.5 \pm 10.4$ \\
\hline Ejection fraction $(\%)$ & 16 & $60.0 \pm 12.5$ & 18 & $56.2 \pm 15.7$ & 18 & $55.2 \pm 10.5$ \\
\hline
\end{tabular}

All values except the number of patients with mitral regurgitation of 1 or less are mean \pm SD. No significant differences were found for each data point. $B P$, Blood pressure; $L A$, left atrial.

of EDV and ESV. Mitral regurgitation was determined with the color Doppler technique. TDI of the mitral annulus was obtained from the apical 4-chamber view. A $1.5-\mathrm{mm}$ sample volume was placed sequentially at the lateral and medial corners of the mitral annulus. Gains were minimized to allow for a clear tissue signal with minimal background noise. Analyses were performed for peak systolic velocity $\left(\mathrm{S}_{\mathrm{a}}\right)$ and early $\left(\mathrm{E}_{\mathrm{a}}\right)$ and late $\left(\mathrm{A}_{\mathrm{a}}\right)$ diastolic velocities. These variables from the lateral and medial annulus were averaged.

Transmitral flow was recorded by placing the sample volume at the tips of the mitral leaflets. From the velocity tracings, early diastolic mitral flow peak velocity (E), late diastolic mitral flow peak velocity (A), deceleration time of the $\mathrm{E}$ wave, and duration of the A wave were measured. The ratio of $\mathrm{E}$ to $\mathrm{A}$ was described as peak E/A. In addition, the color M-mode flow propagation velocity $\left(\mathrm{V}_{\mathrm{p}}\right)$ and the $\mathrm{E} / \mathrm{V}_{\mathrm{p}}$ ratio were obtained with the color Doppler M-mode of the mitral valve.

PV flow velocities were obtained by placing the sample volume 0.5 to $1.0 \mathrm{~cm}$ into the left or right PV. The position of the sample volume was confirmed by obtaining a characteristic PV flow pattern. The Doppler beam was aligned as parallel as possible to the long axis of the blood flow, and the angle correction was within $30^{\circ}$. The peak forward flows during ventricular systole and diastole (S and $\mathrm{D})$ and the height of the retrograde velocity at atrial contraction $\left(\mathrm{A}_{\mathrm{r}}\right)$ were recorded. In addition, the PV flow velocitytime integral (VTI) during ventricular systole was measured by a planimeter from the onset of the forward flow to the onset of the diastolic flow. The onset of diastolic flow was defined as the nadir between the systolic and diastolic waves. The PV diastolic VTI was measured from the nadir between the systolic and diastolic waves to the termination of the diastolic forward wave. The ratio of the systolic to diastolic components was described by peak S/peak D. To maintain consistency and reduce intraobserver variability, echocardiographic evaluations and data analysis of all animals were performed by the same investigator.

\section{Statistical Analysis}

Hemodynamic and Doppler variables were expressed as mean \pm SD. Differences between the mean values for hemodynamic and Doppler variables during the different phases were compared with repeated measures analysis of variance.

\section{Results}

All 19 animals survived the LAA exclusion procedure without any complications and had uneventful postoperative recoveries. When the quality of 2D echocardiographic or Doppler tracings was not sufficient to yield the intended parameters correctly, the image was excluded from the data analyses. The numbers of data points are indicated in Tables 1 through 3 .

\section{Hemodynamic and 2D EE Analyses}

With Doppler imaging, effectiveness of the LAA exclusion was confirmed. Mitral regurgitation was estimated as less than grade $1+$ before and after the LAA exclusion in all animals (Table 1). Hemodynamic data such as heart rate, systemic blood pressure, and mean LAP revealed no significant changes among the data at baseline, after LAA exclusion, or at follow-up. With respect to LA size, a tendency toward a decrease in the LA area or volume was found but was without statistical significance. In addition, there were no significant differences in such 2D EE parameters as EDV, ESV, SV, and EF.

\section{Transmitral Flow, Color M-mode Flow, and TDI Analyses}

Although the E after LAA exclusion was significantly increased relative to those at baseline and follow-up $(P=.001$ and $P=.01$, respectively), there was no difference among 
TABLE 2. Doppler echocardiographic diastolic variables at baseline, after left atrial appendage exclusion, and at follow-up

\begin{tabular}{|c|c|c|c|c|c|c|}
\hline & \multicolumn{2}{|c|}{ Baseline } & \multicolumn{2}{|c|}{ After exclusion } & \multicolumn{2}{|c|}{ Follow-up } \\
\hline & $\mathbf{n}$ & Value & $\mathbf{n}$ & Value & $\mathbf{n}$ & Value \\
\hline \multicolumn{7}{|c|}{ Transmitral flow } \\
\hline$E(\mathrm{~cm} / \mathrm{s})$ & 18 & $41.5 \pm 10.4$ & 18 & $48.2 \pm 10.2^{*}$ & 18 & $41.6 \pm 8.5 \dagger$ \\
\hline$A(\mathrm{~cm} / \mathrm{s})$ & 16 & $34.5 \pm 16.8$ & 17 & $32.2 \pm 13.9$ & 19 & $30.3 \pm 13.1$ \\
\hline E/A ratio & 16 & $1.5 \pm 0.8$ & 16 & $1.7 \pm 0.9$ & 17 & $1.6 \pm 0.5$ \\
\hline \multicolumn{7}{|c|}{ Color M-mode flow } \\
\hline$V_{p}(\mathrm{~cm} / \mathrm{s})$ & 14 & $46.2 \pm 13.2$ & 16 & $44.9 \pm 10.6$ & 13 & $40.0 \pm 9.4$ \\
\hline$E / N_{p}$ ratio & 14 & $1.0 \pm 0.4$ & 16 & $1.1 \pm 0.4$ & 13 & $1.1 \pm 0.5$ \\
\hline \multicolumn{7}{|c|}{ Tissue Doppler imaging } \\
\hline $\mathrm{S}_{\mathrm{a}}(\mathrm{cm} / \mathrm{s})$ & 17 & $7.4 \pm 1.6$ & 14 & $6.7 \pm 1.3$ & 16 & $6.8 \pm 2.1$ \\
\hline $\mathrm{E}_{\mathrm{a}}(\mathrm{cm} / \mathrm{s})$ & 16 & $10.6 \pm 3.9$ & 14 & $10.7 \pm 2.1$ & 16 & $9.8 \pm 3.3$ \\
\hline$A_{a}(\mathrm{~cm} / \mathrm{s})$ & 13 & $9.8 \pm 4.7$ & 9 & $8.3 \pm 2.5$ & 13 & $8.7 \pm 3.8$ \\
\hline $\mathrm{E} / \mathrm{E}_{\mathrm{a}}$ ratio & 16 & $4.5 \pm 1.9$ & 14 & $4.9 \pm 1.4$ & 16 & $4.9 \pm 2.1$ \\
\hline
\end{tabular}

All values are mean $\pm \mathrm{SD}$. $E$, Early transmitral flow velocity; $A$, diastolic transmitral flow velocity; $V_{p}$, color M-mode flow propagation velocity; $S_{a}$ systolic velocity of mitral annulus; $E_{a^{\prime}}$ early diastolic velocity of mitral annulus; $A_{a^{\prime}}$ late diastolic velocity of mitral annulus. $* P<.05$ versus baseline. $† P<.05$ versus after exclusion.

the variables derived from the Doppler transmitral flow and color M-mode flow analyses (Table 2). Furthermore, the $S_{a}$, $\mathrm{E}_{\mathrm{a}}$, and $\mathrm{A}_{\mathrm{a}}$ mitral annular velocities revealed no acute, short-term or midterm changes relative to values at baseline. $\mathrm{The} \mathrm{E} / \mathrm{E}_{\mathrm{a}}$ ratio, an independent parameter of $\mathrm{LV}$ relaxation, also remained consistent without any significant difference.

\section{PV Flow Analysis}

The peak $\mathrm{S}$ velocity at follow-up revealed a significant reduction relative to baseline and after LAA exclusion $(P=$ .0002 and $P=.0005$, respectively) (Table 3 ). The VTI of the $\mathrm{S}$ wave at follow-up had a significant reduction from baseline and after LAA exclusion $(P<.0001$ and $P=.001$, respectively). In terms of the D wave, after LAA exclusion a significant increase in the $\mathrm{D}$ peak velocity relative to baseline was observed $(P=.016)$, whereas the VTI of the $\mathrm{D}$ wave tended to increase but without significant difference $(P=.06)$. At follow-up, the VTI of the D wave was significantly decreased relative to after LAA exclusion $(P=$
.001 ), indicating recovery to the baseline value with $P>.5$ between values at baseline and follow-up.

After LAA exclusion, a significant reduction of the systolic VTI/diastolic VTI ratio was found compared with baseline $(P=.0001)$. At follow-up, there was significant reduction in the peak S/peak D ratio and systolic VTI/ diastolic VTI ratio compared with the data at baseline $(P<$ .0001 and $P<.0001$, respectively).

Each data set of systolic VTI, diastolic VTI, and systolic VTI/diastolic VTI ratio was compared among the three follow-up durations; 7, 30, and 90 days. Traces of these variables, illustrated in Figure 1, show similar progress throughout each of the three follow-up durations. No significant differences were found among the data at 7,30 , and 90 days.

\section{Discussion}

This study revealed significant reductions in the systolic components of the PV flow (peak S and systolic VTI), the

TABLE 3. Pulmonary vein flow velocities at baseline, after left atrial appendage exclusion, and at follow-up

\begin{tabular}{|c|c|c|c|c|c|c|}
\hline & \multicolumn{2}{|c|}{ Baseline } & \multicolumn{2}{|c|}{ After exclusion } & \multicolumn{2}{|c|}{ Follow-up } \\
\hline & $\mathbf{n}$ & Value & n & Value & $\mathbf{n}$ & Value \\
\hline Peak S $(\mathrm{cm} / \mathrm{s})$ & 19 & $36.6 \pm 10.4$ & 18 & $35.6 \pm 15.4$ & 19 & $25.8 \pm 8.9 * \dagger$ \\
\hline Peak D (cm/s) & 19 & $40.7 \pm 11.2$ & 19 & $51.4 \pm 10.7^{*}$ & 19 & $46.9 \pm 14.2$ \\
\hline Peak $A_{r}(\mathrm{~cm} / \mathrm{s})$ & 12 & $18.4 \pm 7.2$ & 10 & $20.3 \pm 4.8$ & 12 & $20.2 \pm 5.6$ \\
\hline Systolic VTI (cm) & 18 & $4.9 \pm 1.2$ & 16 & $4.1 \pm 0.8^{*}$ & 17 & $3.3 \pm 0.7^{*} \dagger$ \\
\hline Diastolic VTI $(\mathrm{cm})$ & 16 & $8.6 \pm 1.8$ & 16 & $9.6 \pm 1.7$ & 18 & $8.2 \pm 0.9 \dagger$ \\
\hline Peak S/peak D ratio & 19 & $0.92 \pm 0.33$ & 18 & $0.75 \pm 0.40$ & 19 & $0.57 \pm 0.19 *$ \\
\hline Systolic VTI/diastolic VTI ratio & 16 & $0.58 \pm 0.14$ & 16 & $0.45 \pm 0.94^{*}$ & 17 & $0.40 \pm 0.09^{*}$ \\
\hline
\end{tabular}

All values are mean \pm SD. Peak $S$, Peak velocity of forward systolic pulmonary vein flow; Peak $D$, peak velocity of forward diastolic PV flow; Peak $A_{r}$ peak velocity of pulmonary vein atrial reversal; VTI, velocity-time integral. $* P<.05$ versus baseline. $\dagger P<.05$ versus after exclusion. 

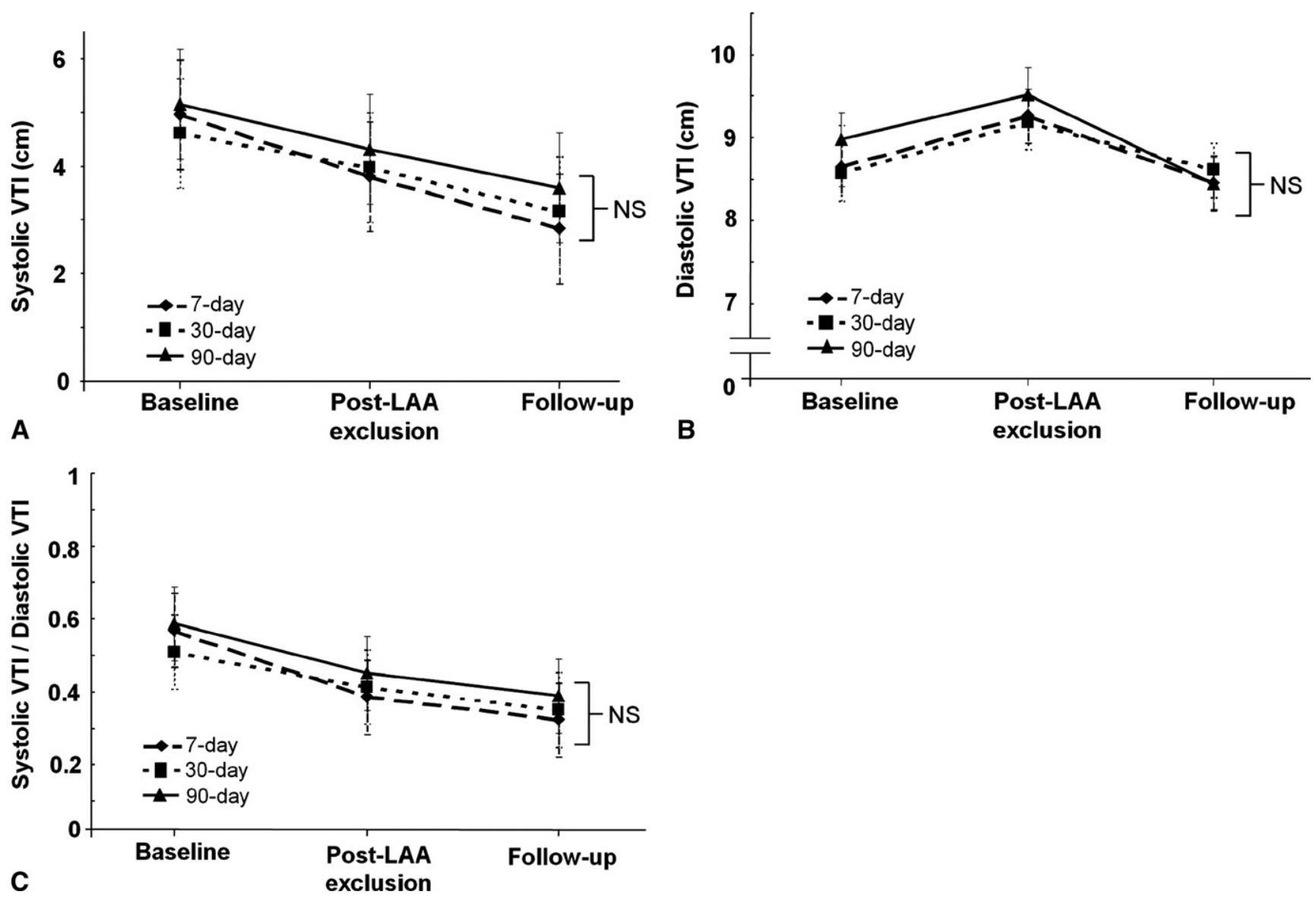

Figure 1. Courses of velocity-time integral (VTI) of forward systolic pulmonary venous flow (A), VTI of forward diastolic pulmonary venous flow (B), and systolic VTI/diastolic VTI ratio (C) during three different follow-up periods (7, 30, and 90 days) after left atrial appendage (LAA) exclusion. There was no significant difference (NS) among follow-up periods.

peak S/peak D, and systolic VTI/diastolic VTI at follow-up after LAA exclusion. Despite these findings, no changes in $\mathrm{E} / \mathrm{A}, \mathrm{E} / \mathrm{V}_{\mathrm{p}}$ and $\mathrm{E} / \mathrm{E}_{\mathrm{a}}$ occurred either acutely or at follow-up. Furthermore, no increase in the mean LAP, which reflects LV filling pressure, was found, indicating that although LAA exclusion profoundly affects LV reservoir function for at least 3 months, the exclusion does not adversely affect $\mathrm{LV}$ filling pressures in a normal heart.

\section{PV Flow Pattern}

The PV flow pattern is determined by transmitral flow and LA function. Considering that transmitral flow can be influenced by LV relaxation and filling, the PV flow is strongly related to $\mathrm{LV}$ diastolic function. ${ }^{12-15}$

The LA function consists of three components: reservoir, conduit, and booster pump functions. In the PV flow pattern derived from the pulsed Doppler analysis, there is a fourwave pattern that correlates with each component of LA function: two forward systolic waves, one forward early diastolic wave, and one reverse late diastolic wave. The first two systolic waves coincide with the LA reservoir phase immediately after LA contraction. The early systolic component is thought to result from LA relaxation, whereas the late component has been described as originating from movement of the mitral annulus toward the apex. The third early diastolic wave occurs during LV relaxation and early rapid filling (the LA conduit phase) and coincides with early transmitral flow. The fourth reverse (into the PVs) late diastolic wave occurs during LA contraction (the LA contraction phase). ${ }^{12-15}$

Influence of LAA Exclusion on LV Diastolic Function Color M-mode Doppler echocardiography and TDI in conjunction with transmitral flow have been shown to provide insight into $\mathrm{LV}$ relaxation and LA function. ${ }^{16-20}$ Several reports have indicated that $\mathrm{E} / \mathrm{E}_{\mathrm{a}}$ and $\mathrm{E} / \mathrm{V}_{\mathrm{p}}$ correlate with $\mathrm{LV}$ 
filling pressures and are reproducible and independent of preload. ${ }^{19,20}$ In this study, $V_{p}$ and $E_{a}$ were measured to assess LV relaxation, and no changes in those variables were observed from the baseline to follow-up. Although LA volume, which is a marker of chronic ventricular overload, ${ }^{26,27}$ was present in 10 dogs, no change in LA area, which shows a strong correlation with LA volume, was observed. In addition, the LAP also remained consistent in all animals. These findings suggest that the LV relaxation was preserved after the LAA exclusion procedure. Because the LAA has been shown to be the most distensible portion of the LA, ${ }^{28}$ an increase in LAP might be expected after LAA exclusion. The constant LAP measurements in this study may be attributable to nonlinear tissue characteristics of the LA, with little resistance to stretch under low pressures, resulting in undetectable increases in the LAP.

\section{Influence of LAA Exclusion on LA Function}

Acute effects. A few previous studies have assessed LA function after LAA exclusion. ${ }^{10,11}$ These reports have demonstrated that LA compliance decreases acutely after LAA ligation or clamping, with decreased LA reservoir capacity and relative increases in the LA conduit function. Our findings support these previous observations. In our study, a significant reduction in the systolic VTI was confirmed, with significant increases in the transmitral $\mathrm{E}$ and the peak $D$ velocities and a tendency toward an increase in the diastolic VTI. These changes in the diastolic components of the transmitral and PV flows may indicate compensatory augmentation of the LA conduit function for impaired reservoir function after LAA exclusion. Because the simple exclusion device allows rapid and easy LAA exclusion in less than 15 seconds, with minimum manipulation to the heart once the LAA is exposed, ${ }^{21,22}$ the interval between the data acquisition points before and after LAA exclusion was relatively brief. Thus all parameters at baseline and after LAA exclusion were compared under similar hemodynamic conditions, such as preloads and afterloads, with the exception of the LAA exclusion.

Short-term and midterm effects. To our knowledge, there have been only a few reports that describe the chronic change in LA function after LAA exclusion. ${ }^{29,30}$ Although Hanna and associates ${ }^{29}$ demonstrated no significant effects on LA function at 6 months after percutaneous LAA occlusion according to PV flow analysis, a tendency toward a decrease in the peak $S$ velocities was found throughout the follow-up durations, which suggests that the lack of significant effects may have been due to the small number of patients $(n=10)$. On the other hand, Isobe and associates ${ }^{30}$ indicated a significant reduction of atrial transport at 6 months in patients with the maze procedure only according to transmitral flow analysis. In our study, the systolic components of PV flow remained in a reduced state at follow- up, which suggests the continuation of impaired LA reservoir function, while the diastolic components of the PV flow and the transmitral E velocity had recovered to baseline values.

The reduction of reservoir function is thought to be due to LA volume reduction by the LAA exclusion, in which the more distensible portion, ${ }^{28}$ the LAA, is isolated from the LA body. One potential factor that influences LA reservoir function chronically, however, may be a restriction of LA movement adjacent to the base of the LAA by an inflammatory reaction in the LA wall that extends from the LAA occlusion site and affects LA distensibility. In this study, there was further significant reduction in LA reservoir function, the peak S velocity and systolic VTI, between after LAA exclusion and follow-up. On the other hand, a comparison of the systolic VTI, diastolic VTI, and systolic VTI/diastolic VTI ratio among the three different follow-up periods revealed no significant difference in each trace of these three variables during each follow-up period. These results suggest that there is no relationship between the degree of LAA reservoir dysfunction and the follow-up period that might potentially influence the degree of inflammatory reaction in the LAA occlusion site. These results further suggest that motion restriction adjacent to the occlusion site has a limited influence on LA reservoir function.

Considering that the results showed no significant difference in hemodynamics, including EF and SV, after the LAA exclusion throughout each study period, LAA exclusion may cause the reduction of LA relaxation in not only short-term but also midterm periods, but may be less influential regarding hemodynamics and cardiac output (CO). Constant LA size and constant LAP measurements also indicate that no increases in LV filling pressures occurred, despite impaired LA reservoir function.

\section{Study Limitations}

None of the animals had AF, so the influence of LAA exclusion on LA function for patients with $\mathrm{AF}$ cannot be discussed. Because Chirillo and colleagues ${ }^{31}$ have described that the PV flow tracing reveals only a diastolic forward component in $34 \%$ of patients with $\mathrm{AF}$, it is possible that the LAA exclusion in patients with AF and compromised reservoir function may have more deleterious effects on the LA reservoir function. Reports that have demonstrated successful application of the Doppler analyses of the PV and transmitral flows in patients with $\mathrm{AF}^{31,32}$ stimulate the need for further studies to evaluate LA function after LAA exclusion with an AF animal model. In addition, considering that patients with AF often have some degree of LV dysfunction with volume overload, an experimental study with our congestive heart failure canine model ${ }^{33}$ could be advantageous to clarify the effects of the LAA occlusion in volume-loaded patients. 
The early and late components of the PV flow during systole were analyzed as a single component because of merging of these components. It is therefore unclear that the reduction of the $\mathrm{S}$ wave is attributable to the impairment of LA relaxation, which may result in the formation of the early component of $\mathrm{S}$ wave. Further evaluation requires an animal model with a low heart rate, so that the $\mathrm{S}$ wave can be discerned as two separate components.

Although compensatory augmentation of the LA conduit function was observed after LAA exclusion, the mechanism to maintain the SV and EF after the recovery of the transmitral E velocity and the diastolic components of PV flow to the baseline remains unclear. Further long-term study is required.

All hemodynamic and echocardiographic data were collected from open-chest preparations, and the dog was under the influence of general anesthesia at each data point. To minimize the influence of mechanical ventilation, however, ventilation was transiently stopped during hemodynamic and echocardiographic data acquisition. Thus the LA data analyses were performed under similar hemodynamic conditions. According to the reports by Hoit and coworkers ${ }^{34}$ and Maniar and associates, ${ }^{35}$ pericardiotomy causes an increase in LA compliance, a reduction in LA contractility, and the loss of the compensatory atrial response after an acute elevation in ventricular afterload. Due to anatomic considerations, the impact of pericardial integrity on LA function is not likely as significant as that on the right atrial function, the lack of pericardial integrity likely affects the PV flow.

\section{Clinical Implications}

Suga and colleagues ${ }^{36}$ demonstrated that a "flexible atrium" (increased receptacle capabilities) would substantially improve the CO. In addition, several reports have demonstrated that pathologically altered LA conduit-to-reservoir function is an important determinant of left heart function and can profoundly affect cardiac performance. ${ }^{12,15,37}$ Therefore the long-term impairment of LA reservoir function may cause reduction in CO. Especially for patients with LV hypertrophy, in which LA function must be maximized, or in patients with $\mathrm{AF}$ and already compromised reservoir function, further careful follow-up after LAA exclusion may be required.

Because of the recent increase in nonvalvular $\mathrm{AF}$ in the aging population, there has been a resurgence of interest in LAA exclusion to reduce the risk of stroke in patients with AF. There is no report, however, that shows clear evidence that LAA exclusion prevents stroke in patients with nonvalvular AF. A randomized trial of LAA occlusion during routine coronary artery bypass surgery (Left Atrial Appendage Occlusion Study) is ongoing and will provide definitive evidence about LAA exclusion in patients without mitral valve disease. ${ }^{7}$ If the LAA exclusion technique is proved to reduce the risk of stroke, a more widespread use of LAA exclusion in patients with paroxysmal AF may be encouraged in the future. Such widespread use raises the concern of LA functional disorder after LAA exclusion. This study indicates that LV parameters (ESV, EDV, CO, and SV) remain unchanged with constant LA area and LAP after LAA exclusion, despite LA reservoir dysfunction, suggesting that LAA exclusion may cause no adverse effects in midterm phase. Considering that LAA exclusion may cause potential adverse effects as a result of reduced production of serum natriuretic peptide, ${ }^{38,39}$ however, further evaluations of not only LA function but also neurohumoral effects after LAA exclusion are definitely required before recommendations can be made.

\section{Conclusions}

LAA exclusion may affect LA reservoir function in the short-term and midterm periods. Further long-term studies with an animal model not only in sinus rhythm but also in $\mathrm{AF}$ or in congestive heart failure are required to fully elucidate the effects of LAA exclusion in patients with impaired cardiac function.

\section{References}

1. Wolf PA, Abbott RD, Kannel WB. Atrial fibrillation as an independent risk factor for stroke: the Framingham Study. Stroke. 1991;22:983-8.

2. Goldstein LB, Adams R, Becker K, Furberg CD, Gorelick PB, Hademenos G, et al. Primary prevention of ischemic stroke: a statement for healthcare professionals from the Stroke Council of the American Heart Association. Stroke. 2001;32:280-99.

3. Stevenson WG, Stevenson LW. Atrial fibrillation in heart failure. N Engl J Med. 1999;341:910-1.

4. Ehrlich JR, Nattel S, Hohnloser SH. Atrial fibrillation and congestive heart failure: specific considerations at the intersection of two common and important cardiac disease sets. J Cardiovasc Electrophysiol. 2002; 13:399-405.

5. Blackshear JL, Odell JA. Appendage obliteration to reduce stroke in cardiac surgical patients with atrial fibrillation. Ann Thorac Surg. 1996;61:755-9.

6. Al-Saady NM, Obel OA, Camm AJ. Left atrial appendage: structure, function, and role in thromboembolism. Heart. 1999;82:547-54.

7. Crystal E, Lamy A, Connolly SJ, Kleine P, Kleine P, Hohnloser SH, et al. Left Atrial Appendage Occlusion Study (LAAOS): a randomized clinical trial of left atrial appendage occlusion during routine coronary artery bypass graft surgery for long-term stroke prevention. Am Heart J. 2003;145:174-8.

8. Davis CA 3rd, Rembert JC, Greenfield JC Jr. Compliance of left atrium with and without left atrium appendage. Am J Physiol. 1990; 259(4 Pt 2):H1356-60.

9. Hoit BD, Walsh RA. Regional atrial distensibility. Am J Physiol. 1992;262(5 Pt 2):H1006-8.

10. Hoit BD, Shao Y, Tsai LM, Patel R, Gabel M, Walsh RA. Altered left atrial compliance after atrial appendectomy: influence on left atrial and ventricular filling. Circ Res. 1993;72:167-75.

11. Tabata T, Oki T, Yamada H, Iuchi A, Ito S, Hori T, et al. Role of left atrial appendage in left atrial reservoir function as evaluated by left atrial appendage clamping during cardiac surgery. Am J Cardiol. 1998;81:327-32.

12. Nishimura RA, Abel MD, Hatle LK, Tajik AJ. Relation of pulmonary vein to mitral flow velocity by transesophageal Doppler echocardiog- 
raphy: effect of different loading conditions. Circulation. 1990;81: 1488-97.

13. Rossvoll O, Hatle LK. Pulmonary venous flow velocities recorded by transthoracic Doppler ultrasound: relation to left ventricular diastolic pressures. J Am Coll Cardiol. 1993;21:1687-96.

14. Appleton CP. Hemodynamic determinants of Doppler pulmonary venous flow velocity components: new insights from studies in lightly sedated normal dogs. J Am Coll Cardiol. 1997;30:1562-74.

15. Barbier P, Solomon SB, Schiller NB, Glantz SA. Left atrial relaxation and left ventricular systolic function determine left atrial reservoir function. Circulation. 1999;100:427-36.

16. Garcia MJ, Ares MA, Asher C, Rodriguez L, Vandervoort P, Thomas JD. An index of early left ventricular filling that combined with pulsed Doppler peak E velocity may estimate capillary wedge pressure. J Am Coll Cardiol. 1997;29:448-54.

17. Nagueh SF, Middleton KJ, Kopelen HA, Zoghbi WA, Quiñones MA. Doppler tissue imaging: a noninvasive technique for evaluation of left ventricular relaxation and estimation of filling pressures. J Am Coll Cardiol. 1997;30:1527-33.

18. Ommen SR, Nishimura RA, Appleton CP, Miller FA, Oh JK, Redfield $\mathrm{MM}$, et al. Clinical utility of Doppler echocardiography and tissue Doppler imaging in the estimation of left ventricular filling pressures: a comparative simultaneous Doppler-catheterization study. Circulation. 2000;102:1788-94.

19. Moller JE, Poulsen SH, Sondergaard E, Egstrup K. Preload dependence of color M-mode Doppler flow propagation velocity in controls and inpatients with left ventricular dysfunction. $J$ Am Soc Echocardiogr. 2000;3:902-9.

20. Nagueh SF, Lakkis NM, Middleton KJ, Spencer WH 3rd, Zoghbi WA, Quiñones MA. Doppler estimation of left ventricular filling pressures in patients with hypertrophic cardiomyopathy. Circulation. 1999;99: 254-61.

21. Kamohara K, Fukamachi K, Ootaki Y, Akiyama M, Zahr F, Kopcak MW, et al. A novel device for left atrial appendage exclusion. J Thorac Cardiovasc Surg. 2005;130:1639-44.

22. Kamohara K, Fukamachi K, Ootaki Y, Akiyama M, Cingoz F, Ootaki $\mathrm{C}$, et al. Evaluation of a novel device for left atrial appendage exclusion: the second generation atrial exclusion device. J Thorac Cardiovasc Surg. 2006;132:340-6.

23. Poutanen T, Ikonen A, Vainio P, Jokinen E, Tikanoja T. Left atrial volume assessed by transthoracic three-dimensional echocardiography and magnetic resonance imaging: dynamic changes during the heart cycle in children. Heart. 2000;83:537-42.

24. Khankirawatana B, Khankirawatana S, Lof J, Porter TR. Left atrial volume determination by three-dimensional echocardiography reconstruction: validation and application of a simplified technique. J Am Soc Echocardiogr. 2002;15:1051-6.

25. Bland JM, Altman DG. Statistical methods for assessing agreement between two methods of clinical measurement. Lancet. 1986;1:307-10.
26. Pritchett AM, Jacobsen SJ, Mahoney DW, Rodeheffer RJ, Bailey KR, Redfield MM. Left atrial volume as an index of left atrial size: a population-based study. J Am Coll Cardiol. 2003;41:1036-43.

27. Moller JE, Hillis GS, Oh JK, Seward JB, Reeder GS, Wright RS, et al. Left atrial volume: a powerful predictor of survival after myocardial infarction. Circulation. 2003;107:2207-12.

28. Hondo T, Okamoto M, Yamane T, Kawagoe T, Karakawa S, Yamagata $\mathrm{T}$, et al. The role of the left atrial appendage: a volume loading study in open-chest dogs. Jpn Heart J. 1995;36:225-34.

29. Hanna IR, Kolm P, Martin R, Reisman M, Gray W, Block PC. Left atrial structure and function after percutaneous left atrial appendage transcatheter occlusion (PLAATO): six-month echocardiographic follow-up. J Am Coll Cardiol. 2004;43:1868-72.

30. Isobe F, Kumano H, Ishikawa T, Sasaki Y, Kinugasa, S, Nagamachi, $\mathrm{K}$, et al. A new procedure for chronic atrial fibrillation: bilateral appendage-preserving maze procedure. Ann Thorac Surg. 2001;72: 1473-8.

31. Chirillo F, Brunazzi MC, Barbiero M, Giavarina D, Pasqualini M, Franceschini-Grisolia E, et al. Estimating mean pulmonary wedge pressure in patients with chronic atrial fibrillation from transthoracic Doppler indexes of mitral and pulmonary venous flow velocity. J Am Coll Cardiol. 1997;30:19-26.

32. Nagueh SF, Kopelen HA, Quinones MA. Assessment of left ventricular filling pressures by Doppler in the presence of atrial fibrillation. Circulation. 1996;94:2138-45.

33. Takagaki M, McCarthy PM, Tabata T, Dessoffy R, Cardon LA, Connor J, et al. Induction and maintenance of an experimental model of severe cardiomyopathy with a novel protocol of rapid ventricular pacing. J Thorac Cardiovasc Surg. 2002;123:544-9.

34. Hoit BD, Shao Y, Gabel M, Walsh RA. Influence of pericardium on left atrial compliance and pulmonary venous flow. Am J Physiol. 1993;264(6 Pt 2):H1781-7.

35. Maniar HS, Prasad SM, Gaynor SL, Chu CM, Steendijk P, Moon MR. Impact of pericardial restraint on right atrial mechanics during acute right ventricular pressure load. Am J Physiol Heart Circ Physiol. 2003;284:H350-7.

36. Suga H, Sagawa K. Instantaneous pressure-volume relationships and their ratio in the excised, supported canine left ventricle. Circ Res. 1974;35:117-26.

37. Prioli A, Marino P, Lanzoni L, Zardini P. Increasing degrees of left ventricular filling impairment modulate left atrial function in humans. Am J Cardiol. 1998;82:756-61.

38. Zimmerman MB, Blaine EH, Stricker EM. Water intake in hypovolemic sheep: effects of crushing the left atrial appendage. Science. $1981 ; 211 ; 489-91$.

39. Stöllberger C, Schneider B, Finsterer J. Elimination of the left atrial appendage to prevent stroke or embolism? Anatomic, physiologic, and pathophysiologic considerations. Chest. 2003;124:2356-62. 